Bulletin du centre

de recherche du

château de Versailles
Bulletin du Centre de recherche du château de Versailles

(2005)

Objets et insignes du Pouvoir

Philippe Kaenel et François Vallotton

\section{Le général et son cheval : figures du pouvoir militaire en démocratie, à l'exemple de la Suisse}

\begin{abstract}
Avertissement
Le contenu de ce site relève de la législation française sur la propriété intellectuelle et est la propriété exclusive de l'éditeur.

Les œuvres figurant sur ce site peuvent être consultées et reproduites sur un support papier ou numérique sous réserve qu'elles soient strictement réservées à un usage soit personnel, soit scientifique ou pédagogique excluant toute exploitation commerciale. La reproduction devra obligatoirement mentionner l'éditeur, le nom de la revue, l'auteur et la référence du document.

Toute autre reproduction est interdite sauf accord préalable de l'éditeur, en dehors des cas prévus par la législation en vigueur en France.
\end{abstract}

\section{revues.org}

Revues.org est un portail de revues en sciences humaines et sociales développé par le Cléo, Centre pour l'édition électronique ouverte (CNRS, EHESS, UP, UAPV).

\section{Référence électronique}

Philippe Kaenel et François Vallotton, « Le général et son cheval : figures du pouvoir militaire en démocratie, à

l'exemple de la Suisse », Bulletin du Centre de recherche du château de Versailles [En ligne], Objets et insignes du Pouvoir, mis en ligne le 20 février 2008. URL : http://crcv.revues.org/114

$\mathrm{DOI}$ : en cours d'attribution

Éditeur : Centre de recherche du château de Versailles

http://crcv.revues.org

http://www.revues.org

Document accessible en ligne sur :

http://crcv.revues.org/114

Document généré automatiquement le 11 octobre 2010.

(c) Tous droits réservés 
Philippe Kaenel et François Vallotton

\title{
Le général et son cheval : figures du pouvoir militaire en démocratie, à l'exemple de la Suisse
}

\author{
«Et l'on fumait du caporal \\ Sous la statue d'un général \\ Était-il, je m'en souviens mal, \\ À pied ou à cheval?» \\ Jean Villard Gilles, La Terrasse des Lilas
}

Fig. 1 Otto Bänninger, Le Général Henri Guisan, 1967, bronze. Ouchy, place du Général Guisan.

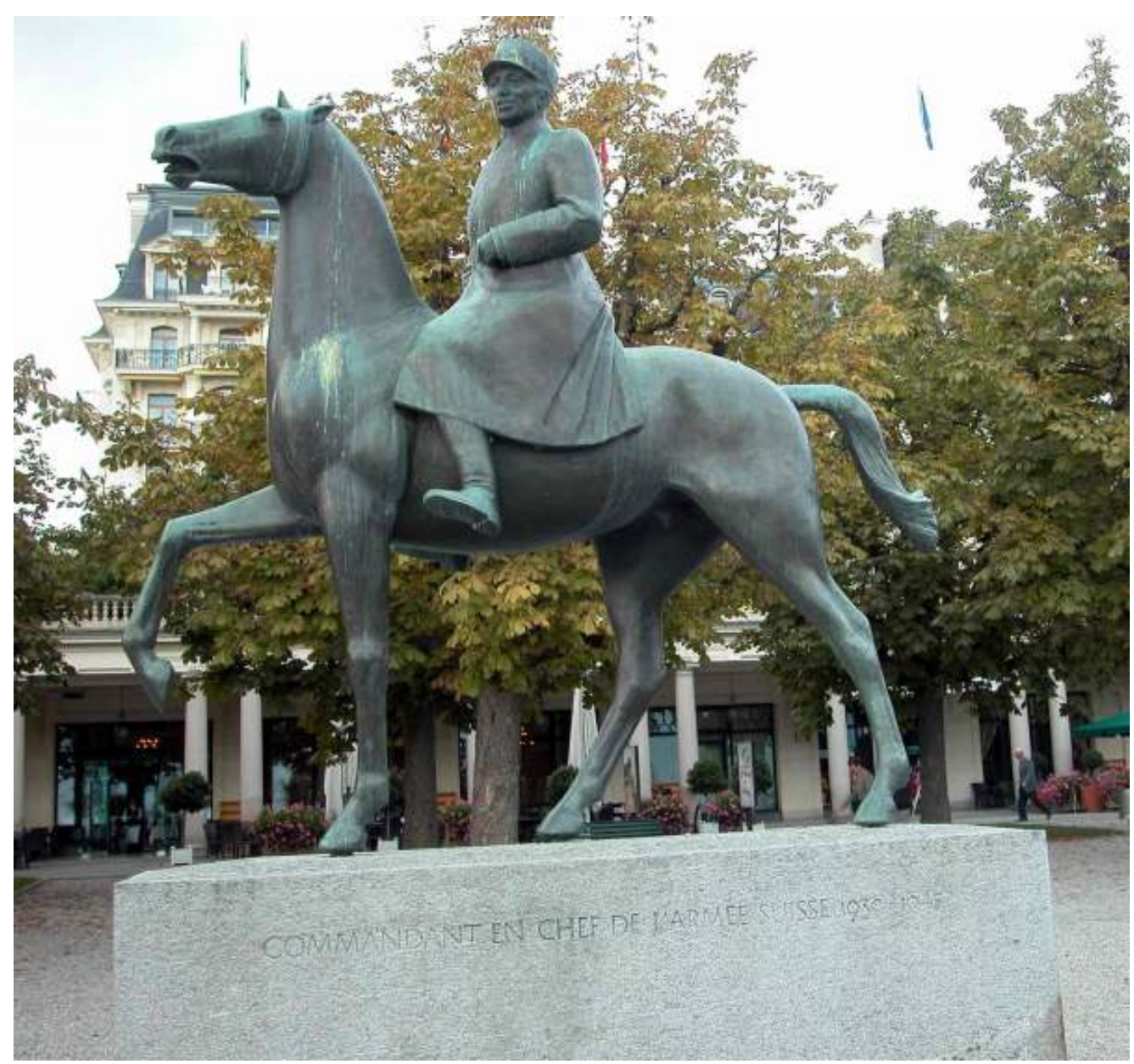

(c) Ville de Lausanne

Le 27 mai 1967 est inauguré, sur le quai d'Ouchy à Lausanne, le monument équestre du général Guisan, le commandant en chef de l'armée suisse pendant la période de la Seconde Guerre mondiale. Cette cérémonie, qui bénéficie d'une couverture médiatique impressionnante, témoigne du caractère quasi mythique de la figure de Guisan dans la culture politique helvétique de l'après-guerre. Elle ne saurait occulter toutefois l'immense polémique dont le monument a fait l'objet depuis la souscription publique lancée pour son édification quelques mois seulement après la mort du général en automne $1960^{1}$. Deux premiers concours débouchent sur autant d'échecs ; puis, le choix du projet du sculpteur zurichois Otto Bänninger 
(fig. 1) provoque la création en 1966 d'un comité « Mouvement national monument général Guisan » qui s'oppose aussi bien à l'emplacement qu'à l'esthétique du monument. Les défenseurs des mânes du héros militaire s'indignent de ce que le monument, initialement prévu au centre ville, soit relégué " hors les murs », dans un endroit présenté comme désert à la morte saison. Par ailleurs, on raille aussi bien le cheval de Bänninger, qualifié de « gazelle dansante », que la posture rigide et martiale du général : «Ce monument est inacceptable. [...] 1. Le cheval ressemble à tout sauf à un cheval. Les allures et les proportions sont celles d'un sujet apocalyptique inconnu chez nous. 2. Le général y est placé d'une façon rigide et martiale qui est absolument contraire à la manière naturelle qui le caractérisait lorsqu'il approchait les soldats et les gens qui l'entouraient. 3. Le général est en manteau. Or tous ceux qui l'ont connu savent pertinemment que jamais le général ne montait en manteau, sauf à quelques rares exceptions $^{2} »$. Le monument du maréchal Mannerheim à Helsinki est souvent cité comme modèle dont on aurait dû s'inspirer. «Mais, conclut un membre du Comité contestataire, si le peuple finlandais a retrouvé son maréchal, le peuple suisse attend son général...». Quelques jours après l'inauguration officielle, un journaliste zurichois de la Neue Zürcher Zeitung, l'organe de référence des élites économiques et politiques helvétiques, prend pourtant fait et cause pour l'artiste : il précise que celui-ci a parfaitement rempli son contrat, à savoir offrir une représentation du général qui, tout en s'inscrivant dans une tradition iconographique classique, est conforme au moment présent et à la spécificité helvétique. Le monument, contrairement aux statues équestres qui trônent sur les places des capitales étrangères, ne joue pas sur le registre de la puissance ou de la domination : le sculpteur s'est au contraire efforcé de rendre la statue plus proche de l'observateur : «Ce général ne monte pas de manière à ce que l'on s'exclame, en le voyant approcher, "un général !" mais de manière à apparaître comme un citoyen parmi d'autres. En ce sens, l'œuvre de Bänninger ne présente pas une image de cavalier dans une perspective traditionnelle, monumentale; elle traduit l'image du "bourgeois", du "Suisse" et transmet ainsi de la manière la plus fidèle le souvenir qu'un peuple reconnaissant veut conserver des hommes et du général Henri Guisan »3.

Cette polémique nous est apparue intéressante à double titre. D'abord elle permet de réfléchir au rôle du cheval en tant qu'emblème du pouvoir au sein des représentations helvétiques. Historiquement, le cheval n'appartient pas au registre symbolique et iconographique auquel est associé le militaire (ou le mercenaire) suisse ${ }^{4}$; depuis le XIII ${ }^{\mathrm{e}}$ siècle, c'est sans conteste l'infanterie qui fait la force des troupes confédérées, une infanterie présente notamment dans les peintures d'un Holbein ou les dessins de Urs Graf. Sur un plan quantitatif, la cavalerie ne représente que $2 \%$ des troupes en 1870 , les mobilisations consécutives à la guerre franco-prussienne révélant en outre une instruction et un équipement largement déficients. Depuis lors, paradoxalement, l'importance symbolique de la cavalerie croît d'autant que son poids stratégique diminue. La Suisse sera d'ailleurs le dernier pays d'Europe à maintenir un corps de cavalerie jusqu'à la décision de sa suppression en $1973^{5}$. Pourquoi cet attachement, de la part des militaires mais également de nombreux citoyens - en 1947, une pétition pour le maintien de la cavalerie recueille plus de 150000 signatures -, à une arme de plus en plus obsolète et quelle place occupe le cheval dans l'iconographie politique et militaire en Suisse ? Sur un autre plan, les représentations du général équestre dans une démocratie comme la Suisse posent problème. Nous verrons que ces représentations, tout en s'inspirant de traditions iconographiques et d'emblèmes du pouvoir transnationaux, ont été réélaborées en fonction du contexte helvétique.

$4 \quad$ Notre étude privilégie les portraits équestres de trois généraux suisses. Précisons que le grade de général est réservé en Suisse au commandant en chef de l'armée en temps de guerre. Depuis la création de la Suisse moderne en 1848, l'Assemblée fédérale a eu l'occasion d'élire quatre généraux : Guillaume-Henri Dufour en 1849, 1856 et 1859, Hans Herzog en 1870-1871, Ulrich Wille en 1914-1918 et Henri Guisan en 1939-1945. Trois d'entre eux se sont singularisés. 
Le Genevois Dufour s'est imposé dans la mémoire collective autant comme le vainqueur de la guerre civile du Sonderbund en 1847 que comme un pacificateur ayant commandé partout à ses troupes de stricts principes d'humanité. Son œuvre de cartographe - contribuant à la construction d'une unité géographique du pays -, ainsi que la part qu'il prend à la création du Comité international de la Croix-Rouge en 1864 ont façonné son image posthume de père de la patrie. Tout autre est le destin d'Ulrich Wille. Associé à l'émergence du militarisme «prussien » à travers ses liens familiaux avec l'Allemagne et ses projets de réforme de l'armée, il est perçu comme une figure autoritaire et germanophile lors de son élection contestée au grade de général en 1914. Henri Guisan, admirateur d'un Mussolini et d'un Philippe Pétain, se révèle quant à lui un utilisateur hors pair des médias modernes qui lui permettront de se présenter en tant que chef charismatique, figure à la fois paternelle et proche du peuple.

\section{Le portrait équestre du général : Dufour comme modèle}

Dans l'histoire de l'art, le portrait équestre s'adresse à trois figures génériques : l'empereur (à l'instar du portrait de Marc-Aurèle sur le Capitole), le chrétien (le miles Christus tel saint Georges terrassant le dragon) et le chef, principalement militaire. Depuis la Renaissance, ses connotations demeurent aristocratiques, notamment dans les républiques oligarchiques qui forment le nord de l'Italie : que l'on songe au portrait peint à fresque de Guidoriccio da Fogliano au Palazzo publico de Sienne (1328), aux deux peintures représentant des monuments équestres au Dôme de Florence (Paolo Uccello, Sir John Hawkwood, 1436 et Andrea Del Castagno, Niccolò da Tolentino, 1455-56) ou encore à la figure canonique de Gattamelata par Donatello à Padoue ainsi qu' au célèbre monument de Verrocchio représentant Bartolomeo Colleoni (Venise, vers 1481-1496). Depuis l'Antiquité, le cheval est ainsi le privilège de l'eques, le noble ${ }^{6}$, même s'il est surtout associé à l'empereur.

D'un point de vue formel, le cheval peut adopter trois postures naturelles, plus ou moins dynamiques : soit il se tient sur ses quatre jambes, soit il en lève une pour évoquer le trot, soit enfin il se cabre en se dressant sur ses jambes arrières ou se lance au galop. Tandis que Giambologna exécute le portrait équestre de Cosimo ${ }^{\mathrm{er}}$ au trot sur le modèle de Marc-Aurèle (Florence, Piazza della Signora, 1587-1599), dans les œuvres du Bernin, de Vélasquez, de Rubens, de Pietro Tacca (Philippe II, Madrid, Plaza de Oriente, 1634-35) ou de Falconet (Pierre Le Grand à Saint-Pétersbourg), les chevaux se cabrent dans un élan baroque. Si ces trois postures (repos, trot, galop) renvoient à l'histoire et aux pratiques de l'équitation, elles n'en demeurent pas moins chargées de significations psychologiques et symboliques déterminantes. Pour comprendre le statut et les intentions du cavalier, il faut aussi regarder sa monture.

À partir du XIX ${ }^{\mathrm{e}}$ siècle, le portrait équestre devient avant tout militaire à l'image de Bonaparte que le peintre Jacques-Louis David immortalise franchissant les Alpes sur un coursier, la crinière animée par le vent de l'Histoire, alors que l'on sait que le général passa le col à dos de mule, comme nous le montre Paul Delaroche en 1850. Dans la Confédération helvétique, la tradition du portrait équestre collectif est établie par le général Guiguer de Prangins, septième commandant en chef de l'armée suisse mobilisée à deux reprises : en 1830 dans la crainte d'une guerre européenne puis en 1838 à l'occasion de l'affaire Louis-Napoléon'. C'est toutefois le général Guillaume-Henri Dufour qui va devenir le point de référence de l'iconographie militaire grâce à sa victoire lors de la guerre civile du Sonderbund en hiver 1847, prélude à la naissance de la Suisse moderne dotée d'une nouvelle constitution démocratique en $1848^{8}$. L'image de Dufour devient rapidement l'objet d'un culte dans la nouvelle Confédération, mais aussi partout en Europe, dans les cercles démocrates. Le général s'en plaint à diverses reprises dans sa correspondance et tente même de lutter contre le phénomène : « Ce déluge, ou plutôt cette grêle de portraits, m'effraie plus que le canon de Gislikon. On veut me tirer de toutes les façons : un artiste me tient dans un morceau de terre, un autre veut mon daguerréotype. On ne 
peut s'en tirer qu' avec un bon portrait qui fasse tomber tous les autres », écrit-il le 6 décembre $1847^{9}$. Il ajoute quelques jours plus tard : « Ces portraits m'assassinent. Mais ce n'est pas tout : il y a maintenant du tabac Dufour, des pipes idem. On fait à Paris des gâteaux Dufour, et cela a l'air d'une mauvaise plaisanterie. Je parais en crème au chocolat... c'est affreux $! »^{10}$. Une telle fortune iconographique est tout à fait exceptionnelle en Suisse. Toutes proportions gardées, dans la diversité de ses supports et ses dérives commerciales, ce phénomène rappelle celui qui entoura Napoléon, le modèle de Dufour.

Fig. 2 Jules Hébert, Le Général Dufour avec son bâton à cheval, 1848, lithographie. Bienne, collection du Musée Schwab.

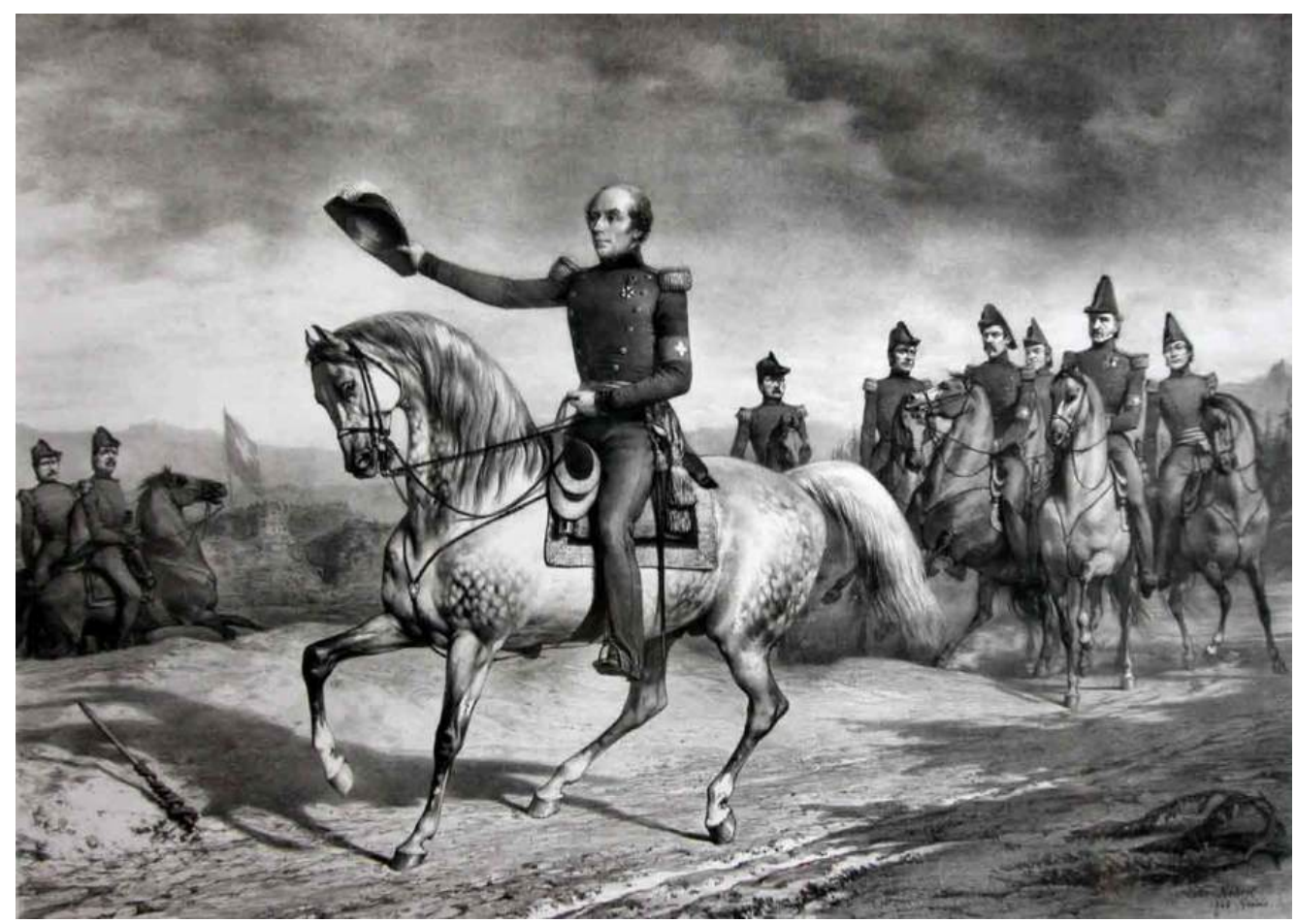

(c) Bienne, Musée Schwab

L'un de ses portraits les plus célèbres est l'œuvre du peintre genevois Jules Hébert (fig. 2). L'artiste présente le général de trois quarts qui arbore le brassard à croix blanche, monté sur un cheval pommelé et piaffant, suivi par l'état-major. À propos de cette lithographie qui ne l'enchante guère, Dufour note : «Si l'on veut absolument faire mon portrait qu'on le fasse bon et sans ostentation. Je préférerais de beaucoup un buste bien dessiné à un portrait équestre, avec d'autant plus de raisons qu'en tout ceci, je suis très peu monté à cheval $»^{11}$. Tout au long de la guerre du Sonderbund et dans les années qui ont suivi, le général a fait montre d'une retenue toute républicaine, quelque peu ébranlée par le culte du héros moderne dont la Suisse n'est pas coutumière, surtout dans un système qui va diviser le pouvoir auprès de plusieurs conseillers fédéraux, empêchant ainsi l'identification du corps à la fonction. Car le culte nouveau de la personnalité s'accommode mal de la vertu républicaine. Dufour tente de résoudre ce dilemme en se présentant non seulement comme la victime de cette imagerie, mais encore comme l'instrument d'une cause plus élevée : « Je ne m'explique ces démonstrations que par la considération que notre lutte a pris les proportions d'une lutte non seulement nationale, mais européenne $»^{12}$. Dufour, en revêtant la figure - notamment équestre - du général qui affiche une modestie républicaine, a établi un modèle dans l'espace public helvétique comme le prouve, un siècle plus tard, la réaction du journaliste de la Neue Zürcher Zeitung face à la statue équestre «bourgeoise » de Guisan inaugurée au bord du lac, à Lausanne (voir plus haut). D'ailleurs, lorsqu'il fut question d'honorer la mémoire de Dufour en 1876, un an après sa 
mort, la question d'un portrait pédestre fut posée car il semblait que cela correspondait plus à l'image républicaine du général. Le sculpteur sélectionné, Karl Alfred Lanz (1847-1907), suivant en cela les désirs du comité, soigna le geste de la main droite qui ne manque pas d'une certaine ambiguïté (fig. 3). Il exprime en effet un mouvement de retenue pacificatrice tout en se calquant sur le modèle du portrait équestre impérial. En d'autres termes, voilà donc un général suisse qui, du haut de son piédestal, montre qu'il a la victoire modeste.

Fig. 3 Karl Alfred Lanz, Projet primé pour le monument du général Dufour, 1884, photographie. Genève, Archive Dufour.

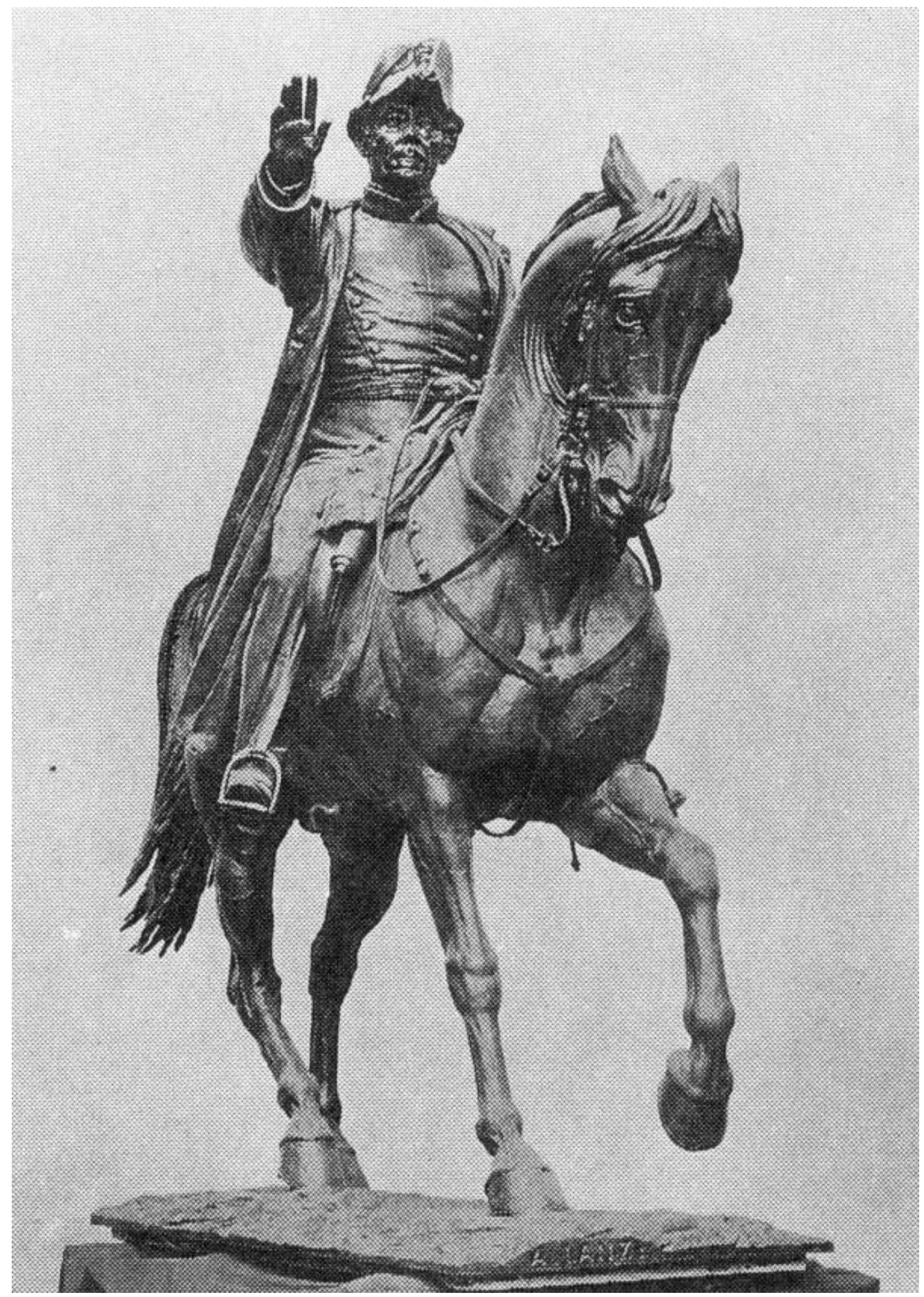

(c) Genève, Archive Dufour 
Jusqu'à Hans Herzog, chef de l'armée suisse pendant la guerre franco-allemande de 1870, le portrait équestre du général est surtout diffusé par la peinture et la gravure. À la même époque, la réforme de l'armée en 1874 et sa centralisation accrue en font un objet central du débat politique, fédéraliste et identitaire qui va s'intensifiant jusqu'à la Première Guerre mondiale. C'est dans ce nouveau contexte que s'inscrit la personnalité du colonel et futur général, Ulrich Wille, qui donne lieu à deux nouveaux registres figuratifs et techniques, attisés par les polémiques : d'une part la caricature, et d'autre part la carte postale photographique.

\section{Ulrich Wille, cavalier teutonique ou gros bourgeois ?}

Fig. 4 Anonyme, Ulrich Wille à cheval de profil, vers 1914, photographie. Berne, Bibliothèque nationale suisse.

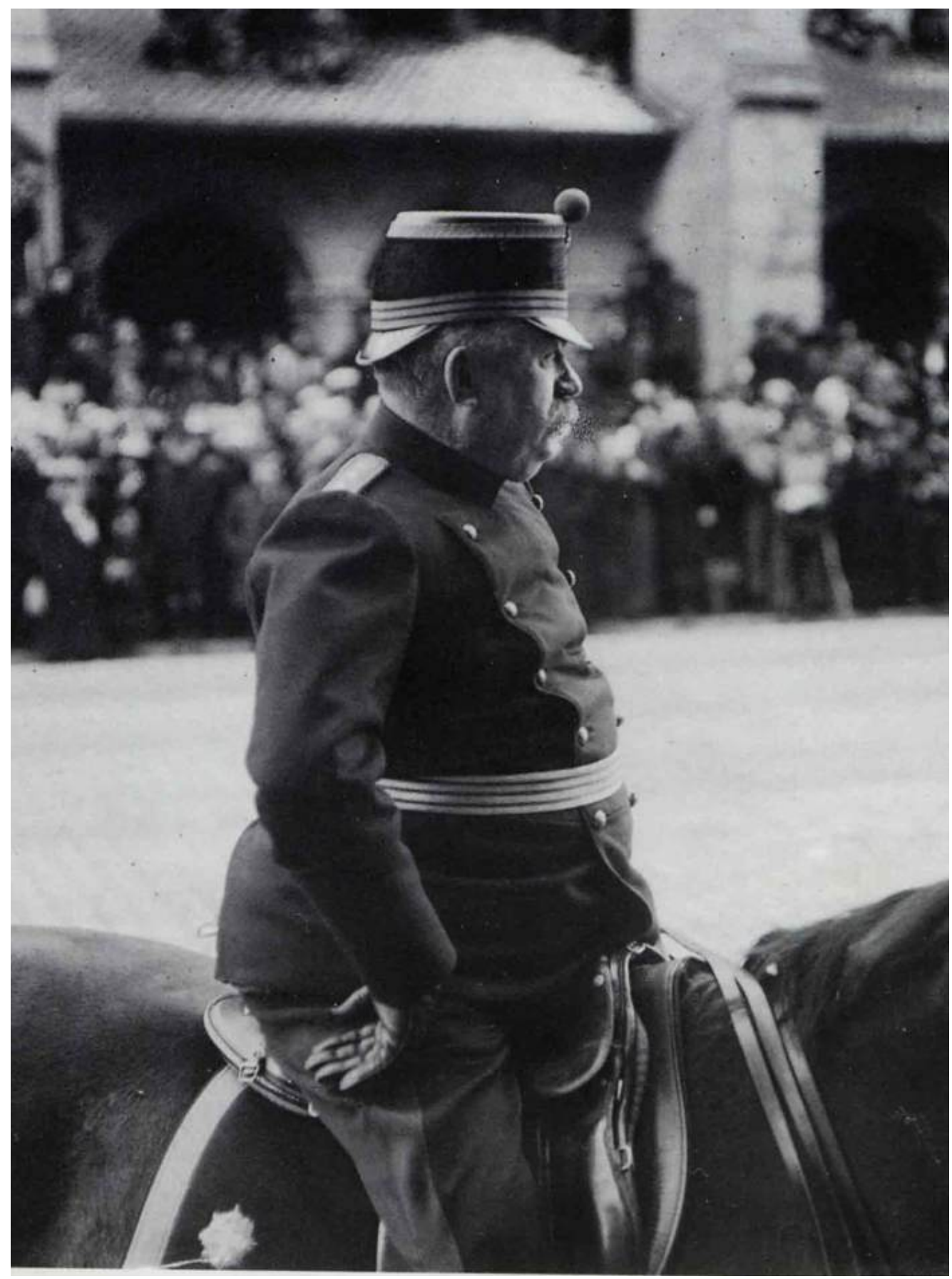

(c) Berne, Bibliothèque nationale suisse 
Fils d'un journaliste appartenant à la vague d'immigration de 1848, Ulrich Wille (fig. 4) étudie le droit à Zurich puis en Allemagne. Lieutenant dans l'armée suisse à dix-neuf ans, il poursuit des études à l'école militaire prussienne de Berlin. Commandant de la cavalerie suisse en 1891, il démissionne de l'armée en 1895 suite au rejet par le peuple des réformes dans lesquelles il s'est engagé. Il se lance alors sans grand succès dans la politique, au sein du parti radical, devient rédacteur de la Revue militaire suisse où il défend des principes prussiens, avant de réintégrer les rangs de l'armée en 1900.

Wille est nommé instructeur en chef de la cavalerie en 1883. Il établit en 1894 un Règlement de Cavalerie, règlement fondateur qui va faire autorité durant la Première Guerre mondiale et bien au-delà. Wille y préconise notamment l'engagement de la cavalerie comme de l'infanterie mobile, un choix tactique qui se heurtera à la vive résistance des officiers de ce corps d'élite ${ }^{13}$. «Élitaire », tel est en effet le mot qui résume la place de la cavalerie au sein de l'état-major et son image publique, même si Wille tente de substituer une logique plus méritocratique aux privilèges de classe qui présidaient à l'intégration dans cette $\operatorname{arme}^{14}$. La cavalerie n'en demeure pas moins le symbole, pour les tenants d'une armée « démocratique », d'un corps qui tient à maintenir, voire à accentuer, son caractère distinctif, tant sur le plan social que symbolique, face à la troupe. À ce titre, il est intéressant de constater qu'au moment où les emblèmes visibles de la hiérarchie militaire tendent à être progressivement éliminés - le hausse-col et les épaulettes sont abandonnés en 1868 alors que le sabre ne joue plus de rôle dans l'instruction -, certains officiers de cavalerie portent, à la veille de la Première Guerre mondiale, la cravache comme symbole de leur position, sorte de substitut du fouet ou du sabre ${ }^{15}$.

Dans ces années, Wille s'impose alors comme l'un des leaders du « Neue Geist », courant qui souhaite une orientation de l'armée suisse vers l'organisation prussienne : plus de discipline, plus d'efficacité, plus d'instruction, et par conséquent plus de drill militaire pour remédier à ses faiblesses. L'image d'un Wille «prussien », renforcée par le mariage du futur général avec une héritière de la famille von Bismarck, se construit dès cette période et resurgira de manière récurrente durant le premier conflit mondial. La personnalité du général Wille est un objet historiographique controversé ${ }^{16}$. Défenseur ou traître à la patrie ? Officier compétent ou sénile ? Les modèles militaires et idéologiques, les réseaux de sociabilité et la correspondance de Wille avec ses proches ne laissent en tout cas guère de doute sur son adhésion à la cause de l'Allemagne prussienne à un moment où la doctrine de la neutralité est érigée en principe politique dominant, imposé auprès des médias de plus en plus polarisés.

Si l'on a beaucoup disserté sur la personnalité de Wille, ses portraits ont peu retenu l'attention, exception faite des peintures que Hodler lui consacre en 1915 et 1916 à l'instigation de Willy Russ, directeur de la fabrique Suchard, et prussien d'origine ${ }^{17}$. La rencontre est exemplaire entre les deux personnalités qui incarnent deux institutions, l'une artistique et l'autre militaire. Wille refuse cette proposition dans un premier temps, répétant le dilemme de Dufour face à son image publique. Wille se déclare certes fier d'avoir été élu à la fonction de général, mais avoue ne pas avoir le profil du chef militaire («Heerführer») car il n'a pas pu en faire la preuve. En effet, tout en étant général, il ne cadre pas avec l'image traditionnelle du chef militaire puisque son rôle politique n'est pas de faire la guerre, mais de l'empêcher sur le territoire helvétique. Le répertoire de ses postures s'en trouve par conséquent limité : portrait en buste, en pied, dans son bureau ou à l'extérieur, inspectant les troupes ou assistant à des défilés. Parmi ses quelques apparitions en peinture, signalons un portrait de groupe intitulé Grenzbesetzung, par le peintre zurichois Johannes Weber en 1914 et 1915. Le général à cheval y figure à la tête de son état-major formé de vingt-quatre individus disposés en éventail devant un paysage avec le lac de Zurich. Un prospectus en propose également la reproduction «artistique » $(71 \mathrm{x} 93 \mathrm{~cm})$ pour le prix assez élevé de six francs. Dans le corpus iconographique du général, la carte postale domine toutefois très largement ${ }^{18}$. Wille y parade souvent à cheval et le cadrage de certaines cartes a pour effet d'accentuer l'embonpoint du personnage (fig. 4). Serait-ce par jeu ou par 
ironie ? Il est difficile de l'affirmer à propos d'un médium populaire et de propagande comme la carte postale. Par ailleurs, notre perception du corps de Wille est faussée par le fait que les cartes postales sont rapidement devenues les sources principales des dessinateurs satiriques, à tel point que le général s'est très vite mis à ressembler à ses charges : une contamination qui est le propre de la caricature comme instrument de contre-propagande visuelle. Contrepoint à la propagande qui se développe incidemment à travers la peinture et la carte postale, la caricature constitue également un phénomène de masse autour de 1900, âge d'or des périodiques illustrés en Suisse. Le principal journal satirique, Der Nebelspalter, fondé en 1875 et édité à Rorschach au bord du lac de Constance, adopte une position prudente quant à Wille. Dans le numéro du 27 janvier 1917, la figure banale du général apparaît à la fois neutre et polymorphe : «Neutralität an höchster Stelle » car, selon le dessinateur du 
Nebelspalter, il ressemblerait tout à la fois au Français Joffre, à l'Allemand Hindenburg, à l'Italien Victor-Emmanuel et à l'empereur François-Joseph d'Autriche.

Fig. 5 Edmond Bille, «Jours de gloire », couverture de l'Arbalète, $n^{\circ} 22,15$ juin 1917, zincographie en couleurs. Lausanne, collection privée.

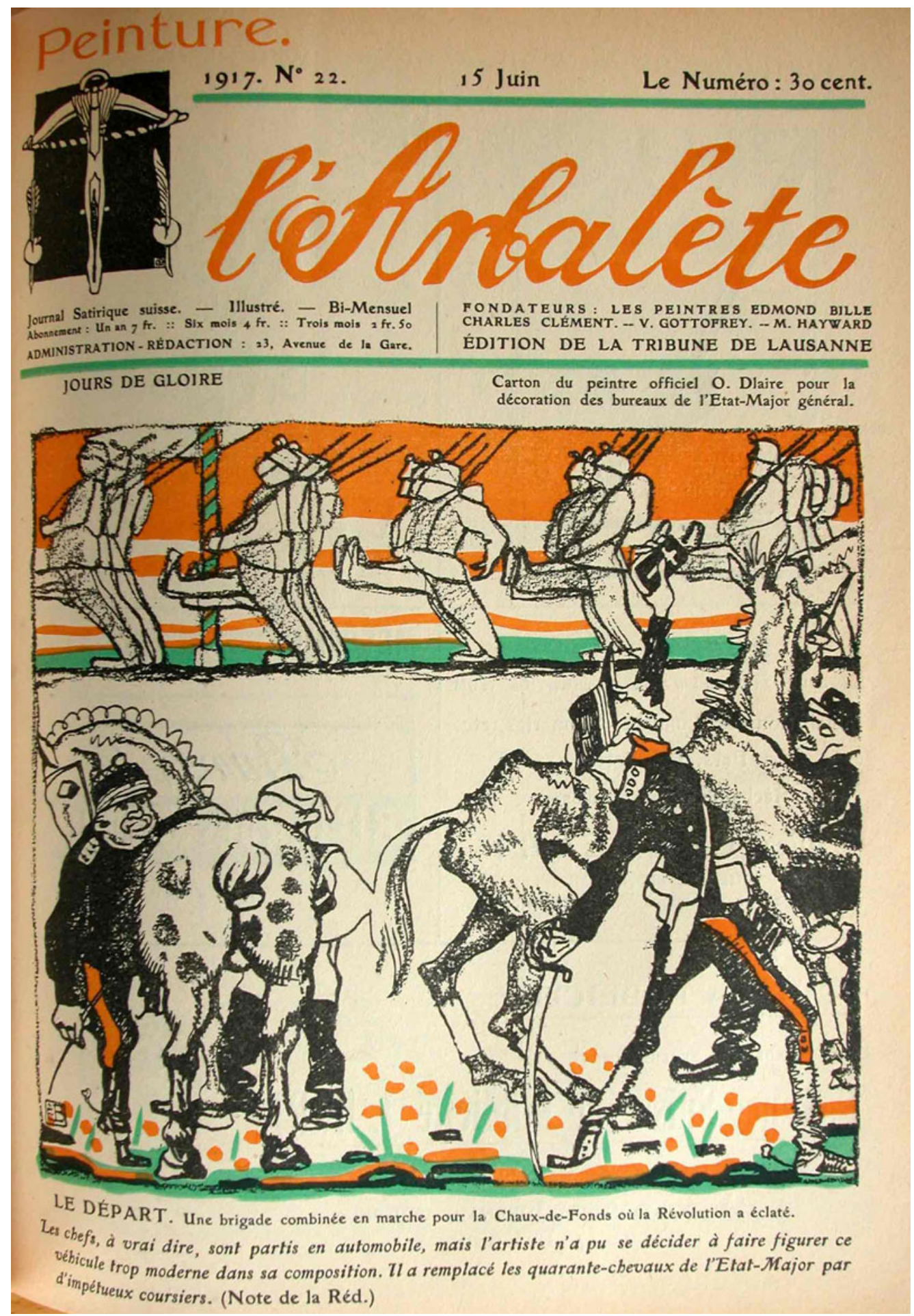

o Archives de la Ville de Lausanne (AVL)

Ces aimables dessins et l'idée d'un Wille «neutre » se distinguent radicalement de l'image qu'en donne la presse satirique suisse romande, et notamment le journal lausannois L'Arbalète (fig. 5), édité par le peintre Edmond Bille en 1916 et 1917. De tendance socialisante, 
ouvertement antimilitariste et ennemi de l'Allemagne, le journal a fait l'objet de pressions de la part de la censure militaire. Wille évidemment devient aussitôt l'une des principales cibles des dessinateurs de L'Arbalète. Ainsi, le numéro de juin 1917 en profite pour faire coup double en visant les deux « chefs » à la fois, soit Wille et Hodler (dont le style et le pouvoir institutionnel sont de plus en plus contestés). Il s'agit d'une parodie du grand tableau commandé à l'artiste par l'Université d'Iéna en 1912, qui représente le Départ des étudiants d'Iéna pour la guerre de libération contre Napoléon en 1813 (fig. 6). Au premier plan, Wille s'apprête à escalader un gros cheval pommelé tandis que son second, le chef de l'état-major Theophil Sprecher von Bernegg (1850-1927) gesticule à côté d'une rosse osseuse. Wille et Sprecher sont en route non pour libérer la nation, comme les étudiants d'Iéna, mais pour réprimer une manifestation populaire à La Chaux-de-Fonds. La légende du dessin d'Edmond Bille tourne en bourrique la fonction traditionnellement héroïque du portrait équestre : « Les chefs, à vrai dire, sont partis en automobile, mais l'artiste n'a pu se décider à faire figurer ce véhicule trop moderne dans sa composition. Il a remplacé les quarante chevaux de l'état-major par d'impétueux coursiers ».

Fig. 6 Ferdinand Hodler, Départ des étudiants d'léna pour la guerre de libération contre Napoléon en 1813, 1908-1909, huile sur toile, léna, aula de l'université.

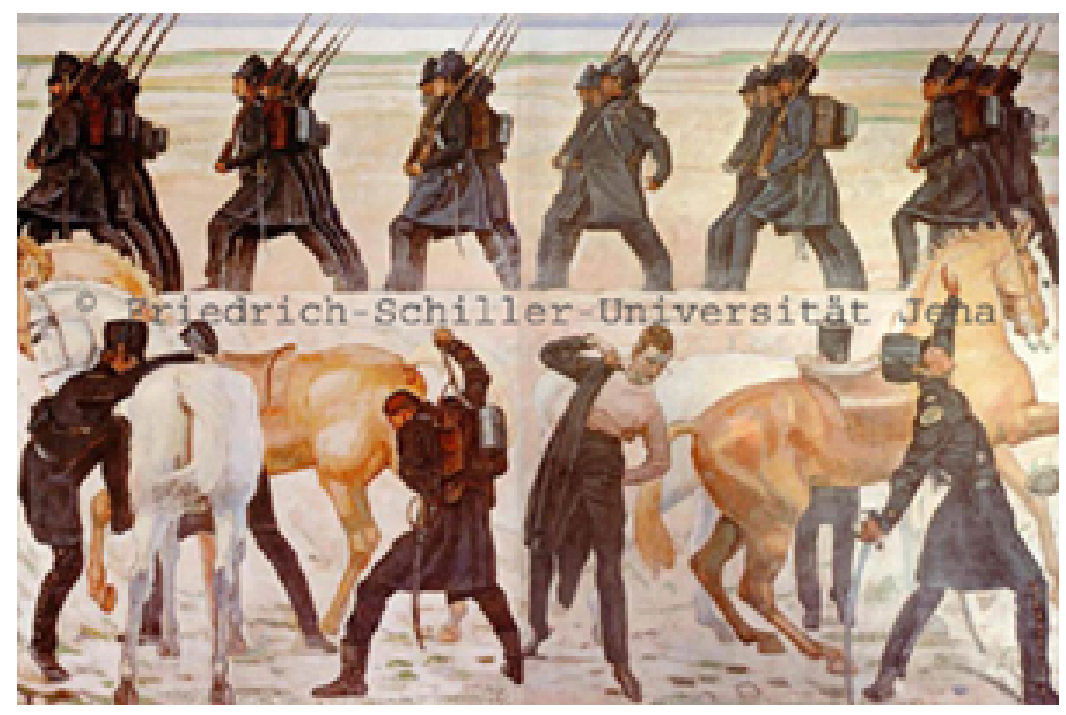

(c) Friedrich-Schiller-Universität Jena

Dans le numéro 8 d'octobre 1916 du journal L'Arbalète, Edmond Bille commente graphiquement la répression militaire d'une manifestation populaire bernoise du 3 septembre (fig. 7). Le très catholique Sprecher est caractérisé par une «Sainte Bible » fixée en bandoulière comme une boîte à munitions, tandis que Wille figure avec son cigare de grand - ou plutôt de gros - bourgeois ${ }^{19}$. Tous deux se présentent de dos tandis qu' au loin, devant eux, la troupe fait face à des moulins à vent d'un rouge éminemment politique. La légende rappelle de manière ironique le rôle de police sociale joué par la milice, et notamment par la cavalerie : « Mais l'armée veillait !... », lit-on au bas de la planche. Assez subtilement, Bille fait ressortir une composante latente de la figure de Wille et notamment du couple Sprecher-Wille. Ce duo présente en effet des traits physionomiques communs avec le duo formé par Don Quichotte et Sancho Pança. D'un point de vue iconographique et physionomique, Wille combine donc les 
traits autoritaires du cavalier teutonique et ceux, prosaïques, d'un bourgeois helvétique dont la posture équestre apparaît tout à la fois incongrue et grotesque, comme celle de Sancho Pança.

Fig. 7 Edmond Bille, «L'aube du 3 septembre 1916 » L’Arbalète, n`8, 15 octobre 1916, zincographie en couleurs. Lausanne, collection privée.

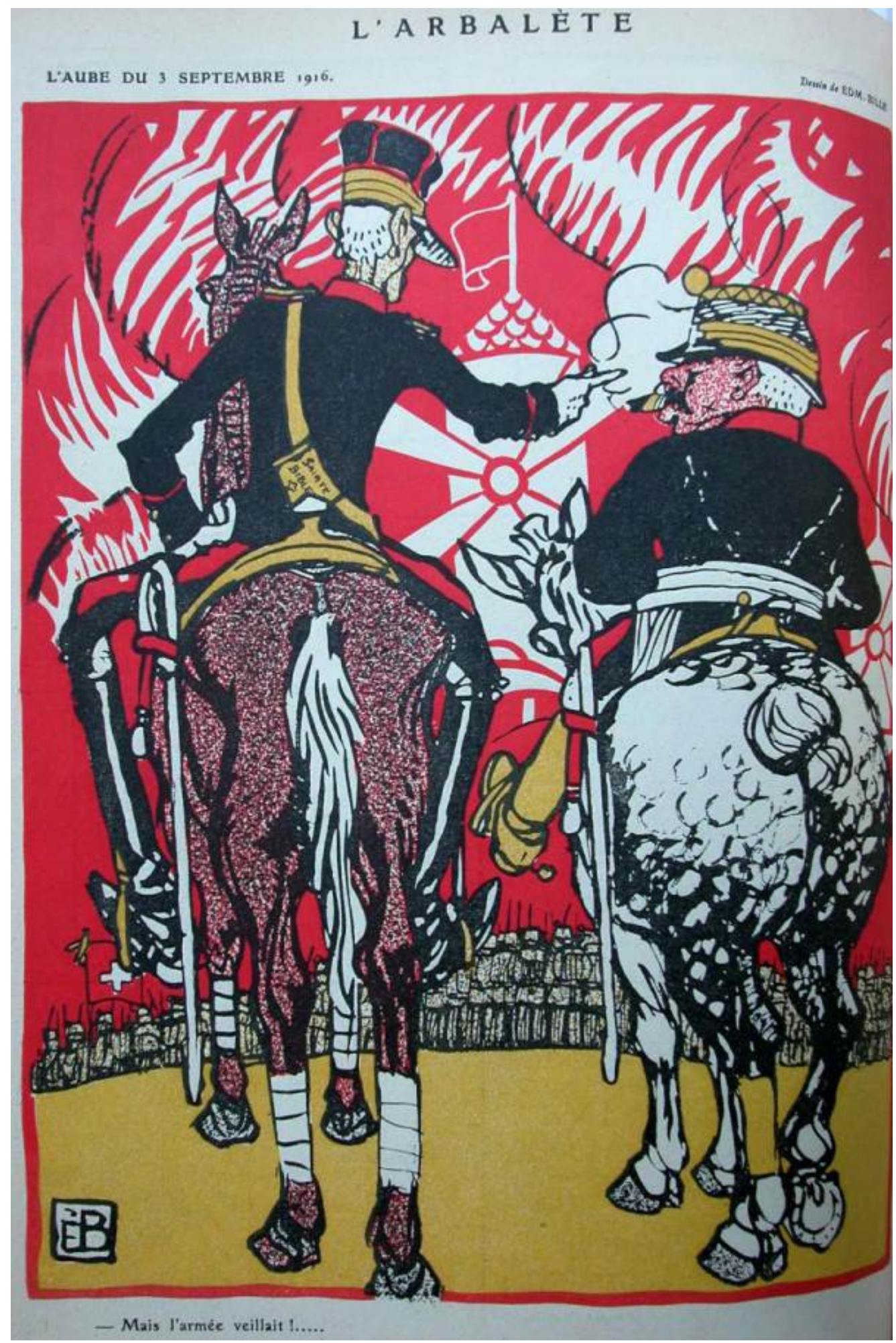

(c) Archives de la Ville de Lausanne (AVL) 
L'image de Wille à cheval manifeste ainsi deux dimensions idéologiques et sociales : d'une part il emblématise le caractère pro-prussien qui caractérise une partie des élites militaires et politiques helvétiques ; d'autre part il illustre le fossé socio-économique qui se creuse dans la société suisse dans ces mêmes années et qui culminera dans la Grève générale de novembre 1918. Quatre ans plus tôt, à l' aube de la Première Guerre mondiale, l'appropriation symbolique du portrait équestre par l'iconographie militaire est indirectement mise en cause à travers la polémique nationale qui se développe lors de l'ouverture de l'Exposition nationale suisse de 1914, autour de l'affiche d'Émil Cardinaux (fig. 8) diffusée à trente mille exemplaires et en trois langues. Abandonnant le registre traditionnel de l'allégorie nationale, l'artiste dessine un jeune paysan qui monte à cru un cheval pommelé. La presse nationale se déchaîne aussitôt autour de ce qui sera dès lors appelé « le cheval vert». «Que signifie, s'il vous plaît, ce petit paysan [...] cet animal découpé dans un bois » (Feuille d'avis de Vevey, 20.9.1913), «grosse cavale habillée d'une peau de truite arc-en-ciel, qui tient du bœuf de l'ours, de l'hippopotame [...] 》 (Le Carillon de Saint-Gervais, 4.10.1913). En fait, au-delà du modernisme (tempéré) de l'affiche, le sujet choisi par Cardinaux détourne le motif héroïque et élitaire du portrait équestre 
dont l'armée vient de s'assurer un monopole symbolique qu'elle s'efforcera de maintenir jusqu'à la Seconde Guerre mondiale.

Fig. 8 Émil Cardinaux, affiche de l'Exposition nationale suisse de 1914, lithographie en couleurs. Berne, Bibliothèque nationale suisse.

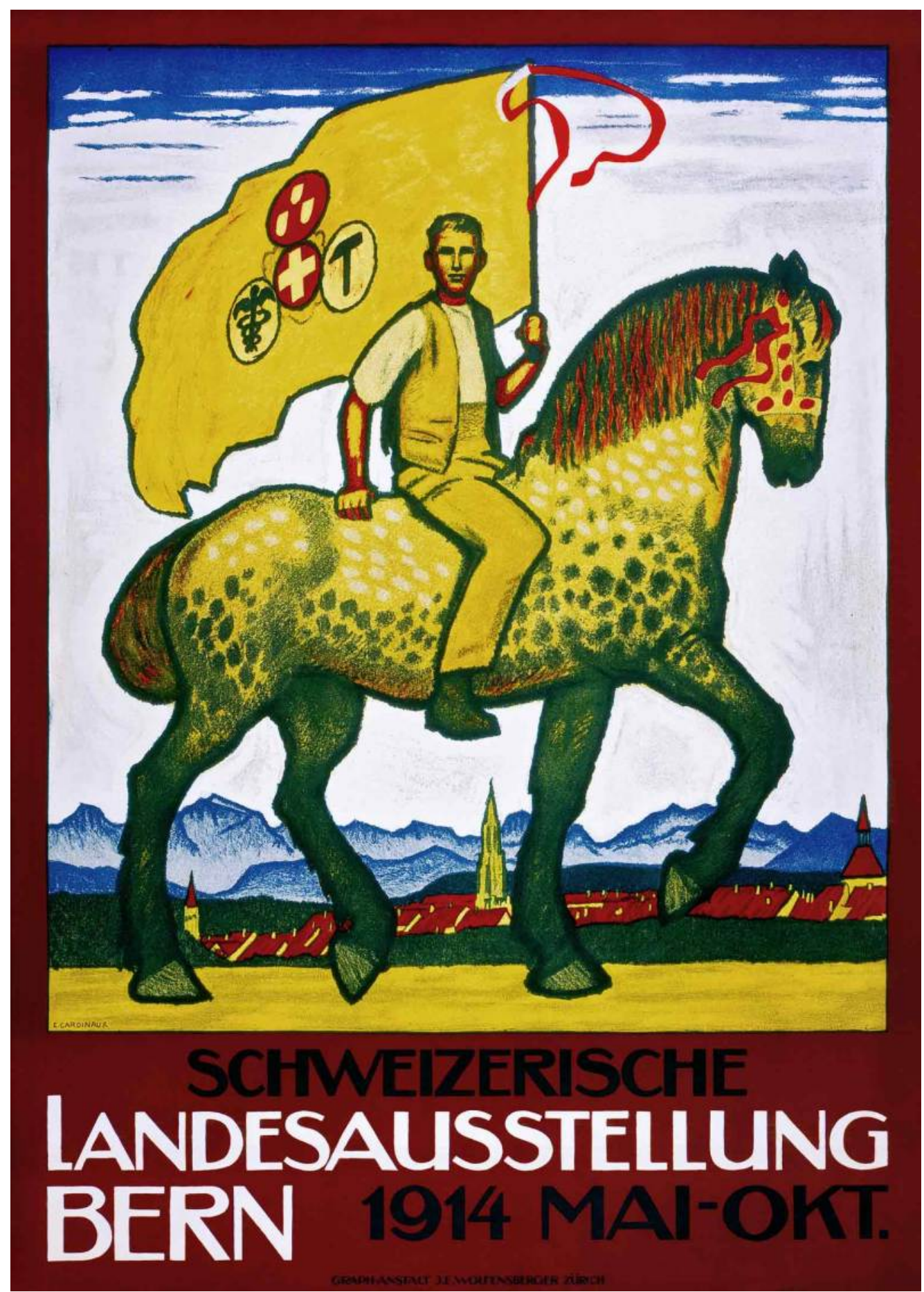

(c) Berne, Bibliothèque nationale suisse

\section{Henri Guisan, gentleman-farmer et officier paysan}

Comment Guisan réinvestit-il le motif du portrait équestre ? Contrairement à son prédécesseur Ulrich Wille, le cheval n'est pas associé à sa formation militaire puisqu'il a fait ses armes en tant qu'artilleur. Mais son rapport au cheval est surtout marqué par un parcours biographique 
qui l'a amené, après des études de droit, à étudier l'agronomie avant qu'il n'acquière un grand domaine dans le canton de Vaud, à Chesalles-sur-Oron. Cette image de gentleman farmer est abondamment cultivée dans l'iconographie de Guisan. On la trouve notamment dans La vie en images du général Guisan du caricaturiste lausannois Gea Augsbourg ${ }^{20}$ (fig. 9), volume publié par Payot en décembre 1939. L'illustrateur vaudois, auteur déjà d'une Vie en images de Darius Milhaud en 1935 et de Serge Lifar en 1937, présente Guisan sous les traits d'un officier-paysan, proche des civils et de la troupe, mais également «proche de son cheval», l'animal étant présenté comme une forme de «totem » du grand homme. À mi-chemin entre la « vie de saint» et l'iconographie populiste à laquelle recourt au même moment le maréchal Pétain (auquel sera envoyé l'ouvrage en janvier 1942), l'album d'Augsbourg recueille l'approbation du principal intéressé. Le dessinateur précise toutefois que cette vision de Guisan ne fera pas l'unanimité au sein de la hiérarchie : un colonel de cavalerie se déclare fâché alors que plusieurs militaristes sont choqués par une vision jugée profanatrice ${ }^{21}$.

Fig. 9 Gea Augsbourg, pages extraites de La vie en images du général Guisan, Lausanne, Payot, 1939, offset. Lausanne, collection privée.

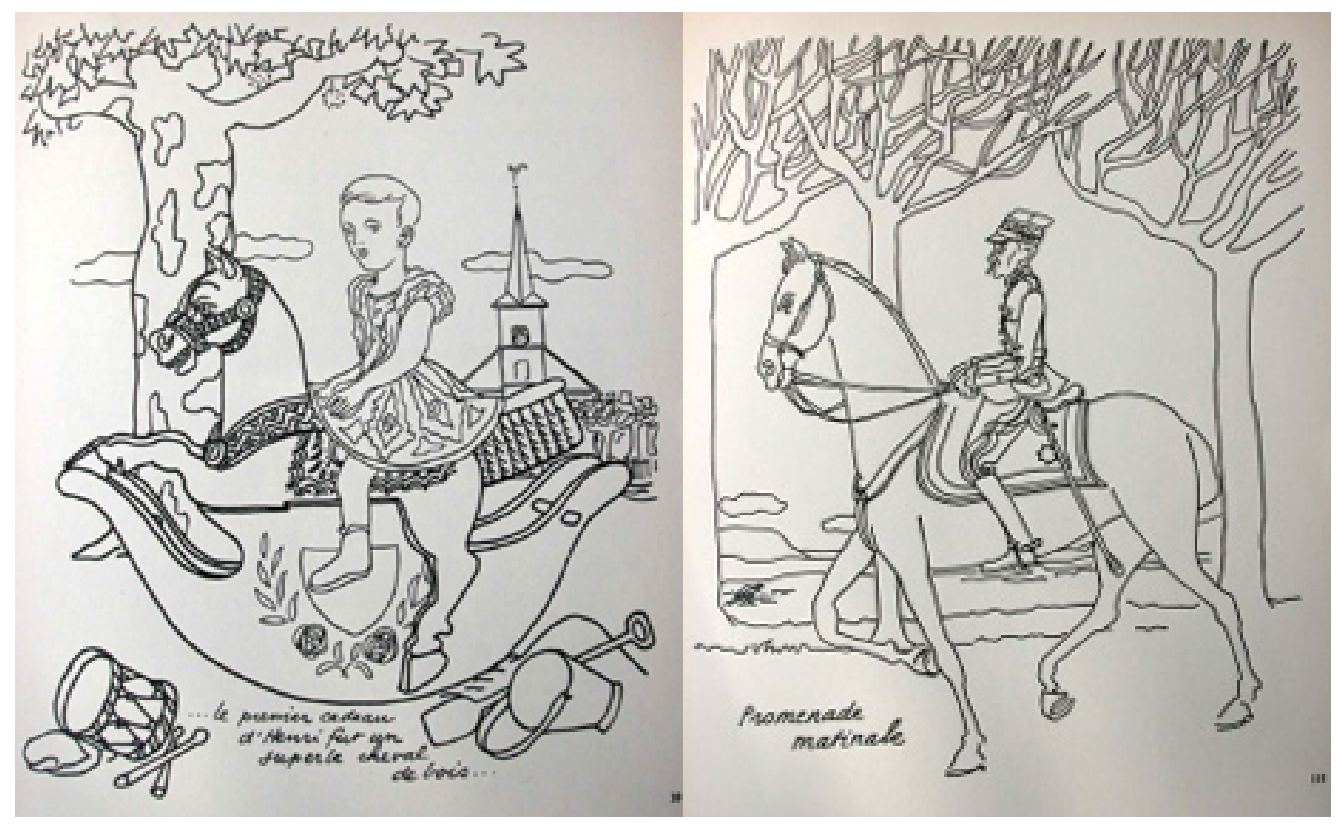

○ Musée historique de Lausanne

Cette image d'un officier-paysan se retrouve dans une séquence du Ciné-Journal suisse de $1944^{22}$ qui présente les activités du général, avec son état-major privé, durant une journée. On peut y voir Guisan partir en promenade avec son fidèle destrier, nommé Nobs. La proximité cavalier-cheval est ici renforcée par l'usage d'un patronyme, une pratique que l'on retrouvera pour toutes les montures du général. Après avoir donné à son cheval sa friandise matinale (une carotte), Guisan est filmé galopant à travers champs et bottes de paille, puis, toujours en selle mais autour d'une fontaine, en train de discuter - en allemand - avec un paysan. Le cheval n'apparaît pas ici comme emblème du pouvoir ou marque de distinction militaire mais comme signe d'une appartenance sociale - la paysannerie - et incarnation d'une culture rurale, abondamment mise en valeur par ailleurs dans le contexte de Défense nationale spirituelle, mais aussi dans la propagande pétainiste par exemple. Le cheval témoigne également de la dimension très populiste de ce documentaire, apparente dans le bref aparté entre le général et le paysan. Le cheval est moins ici un emblème de différenciation sociale ; il sert plutôt comme élément de rapprochement, d'ancrage, voire de communion avec la population. Enfin, la scène - tout comme le dessin d'Augsbourg - évoque une promenade et non une parade. L'épisode ressortit plus au registre agreste, voire sportif, que militaire. Il souligne d'abord 
le caractère fringant et athlétique d'un personnage qui compte soixante-cinq printemps au moment de sa nomination en tant que commandant en $\operatorname{chef}^{23}$. Sur un autre plan, il renvoie à l'une des particularités de Guisan, à savoir le soin qu'il mettait à se donner comme représentant et incarnation du peuple tout entier, et non de l'armée uniquement.

En effet, Guisan a tout spécialement veillé à la construction de son image publique. Une des premières décisions d'importance du général au lendemain de sa nomination est de se constituer un état-major personnel, sorte de cabinet parallèle chargé de l'assister dans son commandement. Cet état-major privé, qu'il établit au bord du lac de Brienz puis à Interlaken (dans les Alpes bernoises), prête une grande attention à sa communication, que ce soit par l'écrit, le son ou l'image. Comme le précise l'historien Willi Gautschi, «l'état-major particulier fit preuve d'une grande inventivité en faveur de la position et de la personne du commandant en chef, dans la création et l'exploitation de canaux propres à engendrer un effet psychologique plutôt inhabituel pour les concepts suisses ${ }^{24} \gg$. Le bureau de presse de l'état-major général s'est également s'appuyé sur de nombreux reporters, photographes et cinéastes. Placé sous la responsabilité des services de renseignements avec pour mission d'informer directement la population et la troupe, le bureau dispose de son propre organe de censure, particulièrement attentif aux représentations qui s'écarteraient des présentations agréées de Guisan. On estime ainsi que sur 25000 clichés rapportés par les photographes, près de 5600 ont été interdits de publication ${ }^{25}$.

Fig. 10 Otto Baumberger, Nos chefs d'Armée, 1939, lithographie en couleurs. Berne, Bibliothèque nationale suisse.

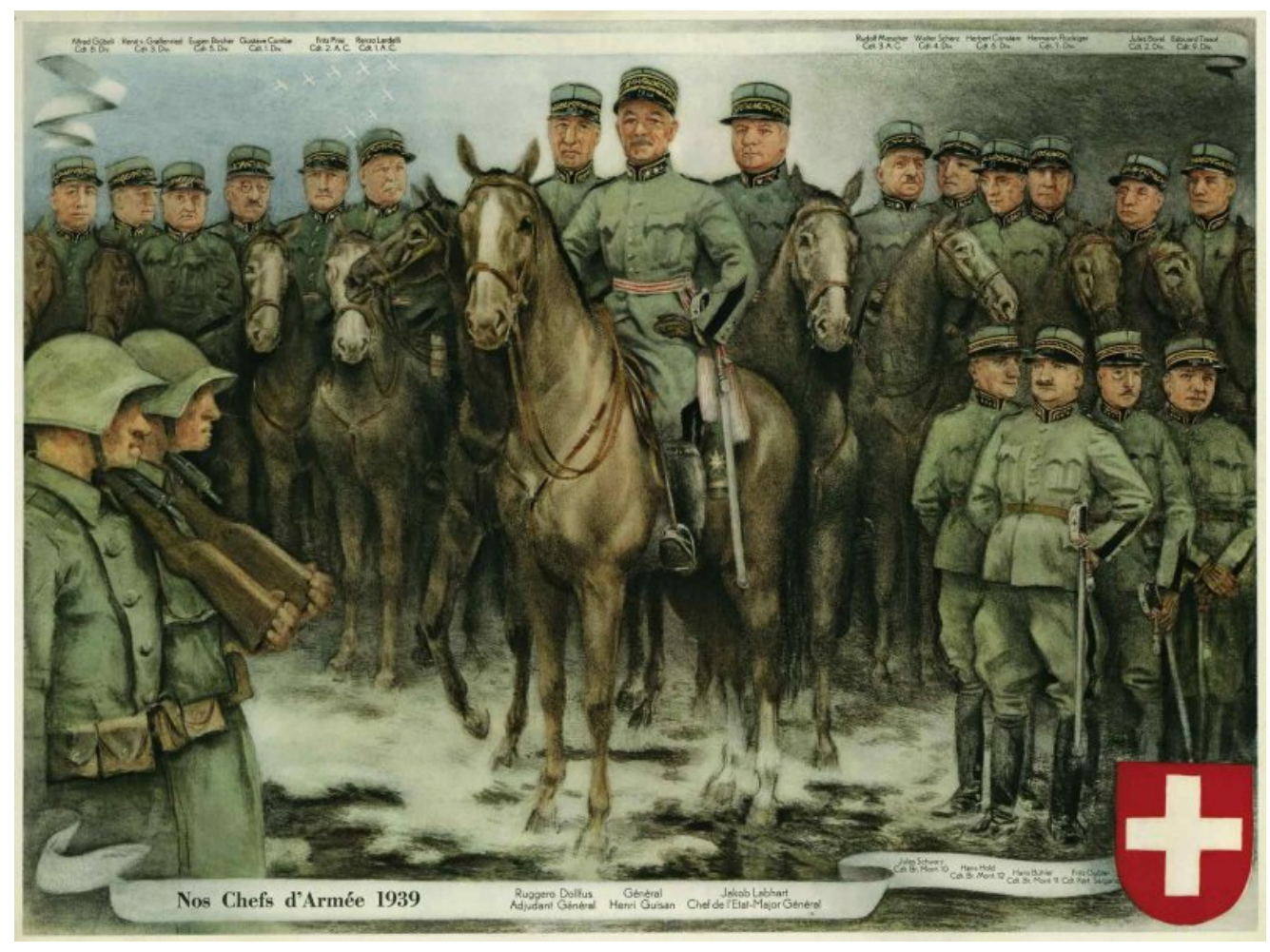

(c) Berne, Bibliothèque nationale suisse

Parmi ces images censurées, la lithographie d'un portrait équestre collectif a connu un sort particulier. Otto Baumberger, illustrateur et affichiste suisse de renom mais aussi collaborateur du journal satirique d'audience internationale le Nebelspalter, réalise en 1939 une sorte de portrait de groupe de l'état-major (fig. 10). Ce tableau n'a pas l'heur de plaire au général : son état-major personnel intervient en effet afin d'interdire la vente et la diffusion de la planche, l'édition étant par ailleurs détruite à l'exception d'un petit nombre d'épreuves. L'œuvre est envoyée au «purgatoire » (l'antichambre de l'Enfer) de la Bibliothèque nationale, où elle 
restera jusqu'en février $1949^{26}$. Cet épisode témoigne d'abord de l'extrême vigilance de Guisan quant à la gestion de son image. Plus fondamentalement, le général récuse ici un certain ridicule de la représentation dû entre autres à la disproportion entre la taille des hommes et celle des chevaux et au caractère impossible de ce portrait de groupe : une dimension qui sera accentuée par le trait humoristique du graphiste et affichiste neuchâtelois Éric de Coulon dans une parodie intitulée « Le revers de la médaille... ou les trucs dévoilés » (fig. 11), qui montre la même scène vue de dos. Mais surtout Guisan ne veut se reconnaître dans cette vision à la fois «pompière » et officielle qui présente l'état-major comme une forme de « caste », totalement figée et déconnectée de la société.

Fig. 11 Éric de Coulon, Le Revers de la médaille... ou les trucs dévoilés, 1939, lithographie. Berne, Bibliothèque nationale suisse.

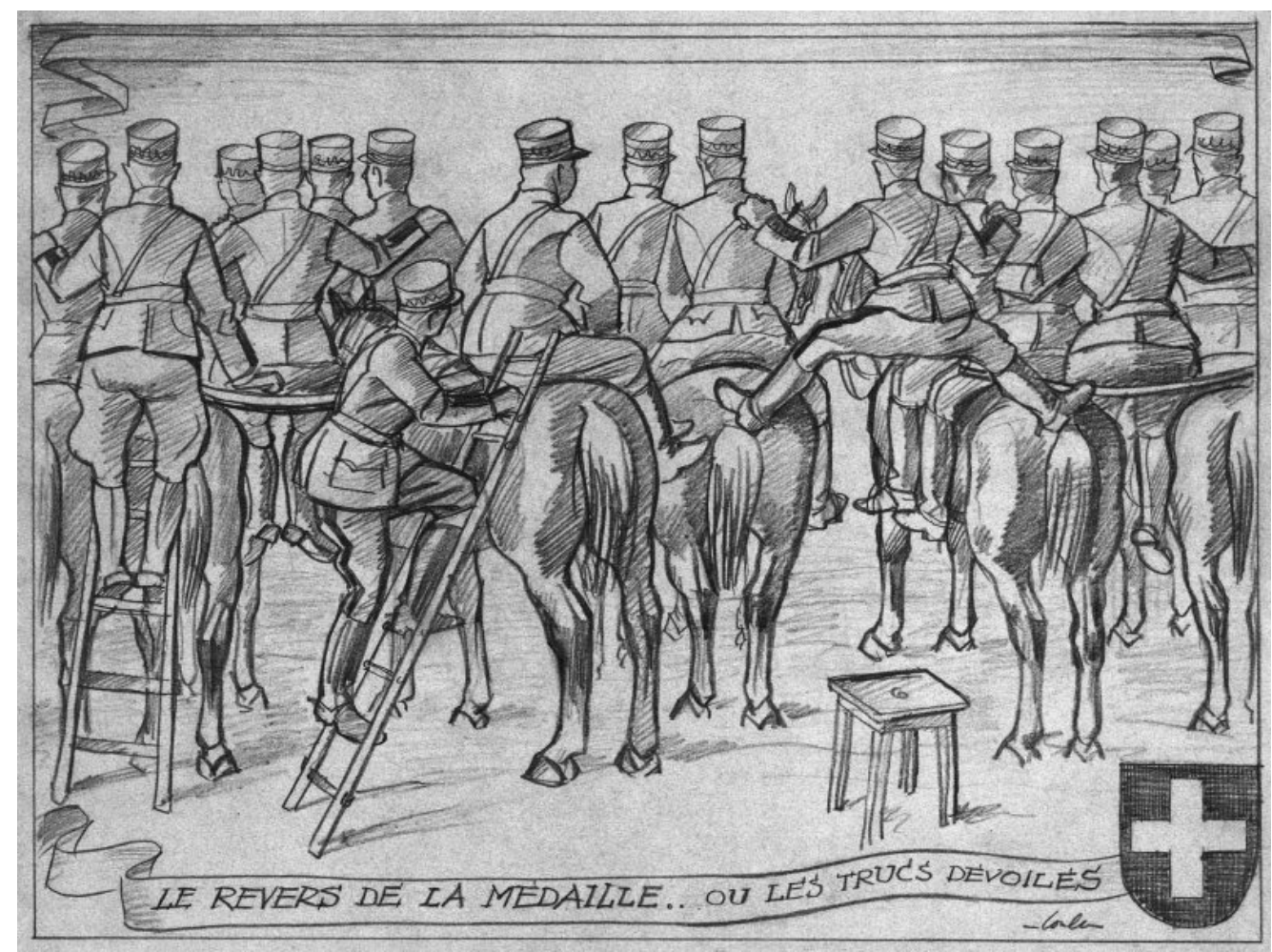

(c) Berne, Bibliothèque nationale suisse

Dans toutes ses représentations, qu'elles soient picturales, photographiques ou cinématographiques, Guisan veille avec le plus grand soin à apparaître comme une forme de leader naturel, et non comme l'incarnation d'un corps ou d'une autorité exclusivement militaire. L'icône d'un chef charismatique, proche de la terre et du peuple, est ainsi relayée à outrance par les différents médias de l'époque : illustrés, actualités cinématographiques, radio, voire produits dérivés. Une image qui a longtemps réussi (et qui réussit encore largement aujourd'hui) à masquer les penchants autoritaires d'un personnage, grand admirateur du fascisme italien, et qui n'hésite pas à outrepasser les pouvoirs qui lui ont été conférés en prenant, en plusieurs circonstances, différentes initiatives, à l'insu du collège exécutif fédéral ${ }^{27}$.

\section{Démocratie et dénégation}

La photographie de Guisan devant le monument du général Dufour, prise lors d'une parade militaire de 1943 (fig. 12), résume les enjeux de la figure équestre du général en Suisse. Le 
parallèle et le vis-à-vis explicite entre les deux personnages sur leurs montures placent Guisan dans le lignage symbolique d'un chef militaire exemplaire aux yeux de l'historiographie.

Fig. 12 Anonyme, Le général Guisan sur la place Neuve à Genève devant le monument Dufour, 1943, photographie. Pully, Centre général Guisan.

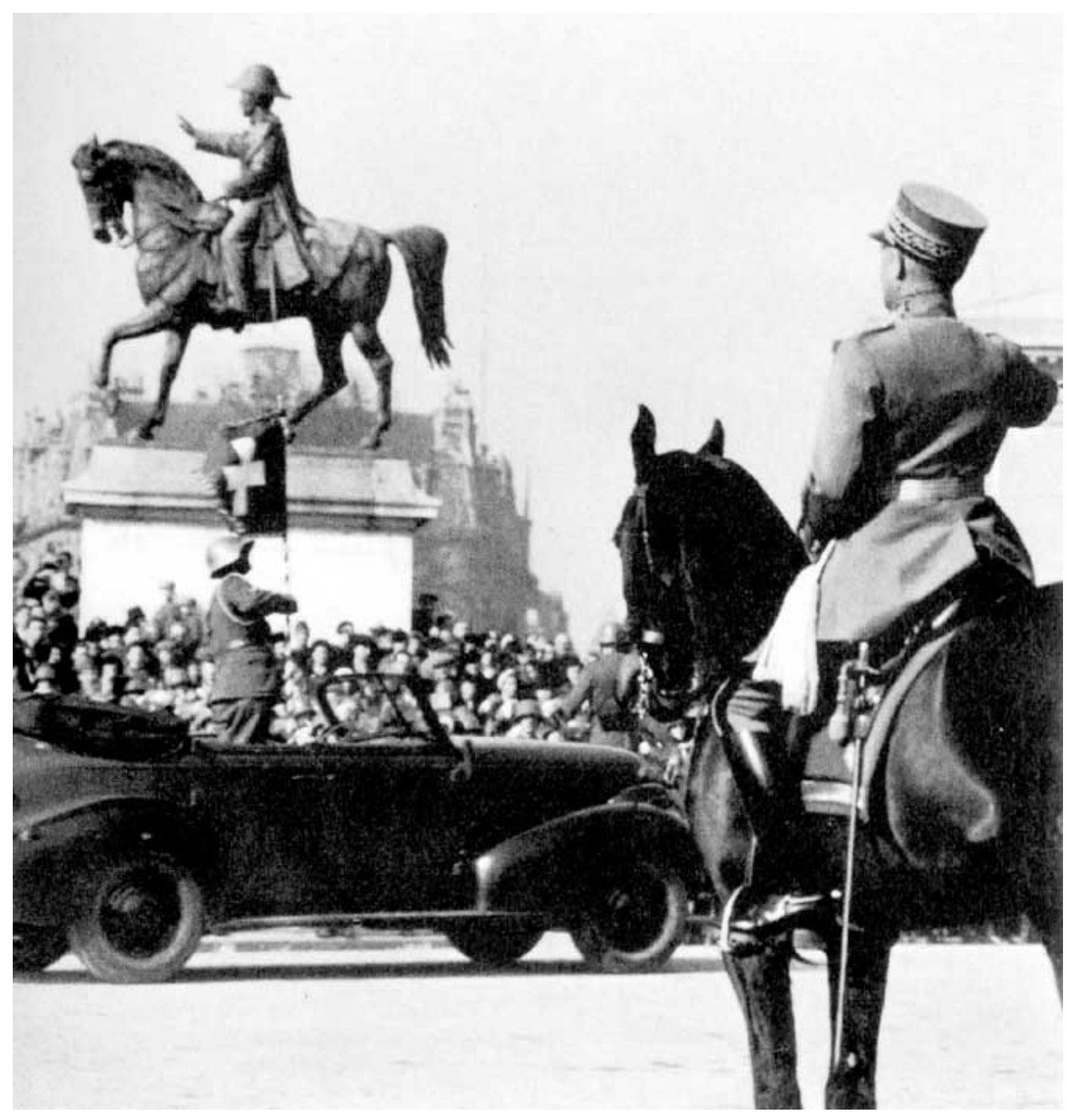

○ Pully, Centre général Guisan

À priori, tout semble pourtant opposer les deux hommes dans leur manière de concevoir leur fonction et de gérer leur image publique. Dufour déteste les représentations guerrières et récuse les images qui le présentent dans des postures par trop agressives ou ostentatoires. Il ne peut dissimuler son agacement devant la multiplication mercantile de son image dans d'innombrables produits dérivés qui, outre leur dimension mercantile et leur facture caricaturale, génèrent une forme de culte hérö̈que qui lui semble en décalage avec le sens de son action. Guisan au contraire prête un soin minutieux à construire une image publique qui cultive un certain prestige, voire une forme d'autoritarisme, tout en évitant le reproche d'élitisme. Guisan, dont la vie et la carrière sont un exemple de réussite sociale en démocratie, en bref ce général si populaire, ne se prive pas en effet de recourir à des postures ou des représentations étroitement reliées au « culte du chef » tel qu'il se développe dans les régimes autoritaires à la même époque.

Dans le même temps, tous deux entretiennent un rapport ambigu au genre du portrait équestre. Dufour le récuse car trop éloigné de son esprit républicain. Dans une lettre à sa fille, il précise que si l'on veut absolument faire son portrait, il préférerait de beaucoup un buste bien 
dessiné à un portrait équestre ${ }^{28}$. Chez Guisan, la posture équestre souligne un attachement à des valeurs traditionnelles et rurales qu'il décline notamment en ouverte opposition à l'essor de l'urbanisation, à l'industrialisme de même qu'au socialisme. De plus, au moment où l'armée suisse est amenée à jouer un rôle idéologique inversement proportionnel à sa fonction stratégique et militaire, le cheval devient l'emblème d'un peuple fier de ses traditions et soudé autour de valeurs communes.

$\mathrm{Au}$ terme de ce parcours, nos trois généraux à cheval expriment une sorte de dénégation de la figure équestre traditionnelle. Cette dernière renvoie en effet d'une part à une forme d'autoritarisme contraire à la tradition et aux institutions helvétiques, d'autre part à une attitude héroïque en décalage avec la vocation d'un État neutre, non belligérant. Cette dénégation se décline selon divers registres. Chez Dufour, c'est le rejet de la représentation équestre elle-même ou, pour son sculpteur, l'invention d'une posture qui emprunte aux statues impériales classiques tout en lui conférant - avec le geste du bras droit évoqué plus haut - une retenue pacificatrice. Chez Wille, c'est l'absence d'un monument équestre qui est significative : un tel monument aurait sans doute été inacceptable par la population dans la mesure où il aurait accentué l'image autoritaire et hautaine du personnage tout en ravivant les plaies occasionnées tant par le fossé entre Suisse allemande que Suisse romande que par la Grève générale de 1918. Le Wille cavalier est avant tout la cible des caricaturistes. Quant à Guisan, sa position est la plus ambiguë. Construisant une grande part de son aura sur le registre symbolique, Guisan s'efforce de soigner son image de « conducator » tout en soulignant sa proximité avec la population et en récusant les poses et attitudes par trop martiales. Une attitude confirmée par le témoignage de son chauffeur livré à l'occasion de la polémique autour du monument équestre d'Ouchy vers 1966 : «C'est ainsi qu'il [le général] parlait parfois du monument qui s'érigerait vraisemblablement après sa mort comme ce fut le cas des autres généraux suisses. J'ai encore présentes à la mémoire ses paroles à ce sujet : Voyez-vous, Weissbrodt, j'espère que le ou les sculpteurs sauront éviter le genre martial, "hoch zu Ross" comme disent nos compatriotes. J'ai servi une armée, un peuple avant tout. 
Puissent les sacrifices de toutes les Suissesses, de tous les Suisses, être avant tout rappelés aux générations futures ${ }^{29} \gg$.

Fig. 13 Anonyme, Les funérailles du général Guisan à Lausanne en avril 1960, photographie. Pully, Centre général Guisan.
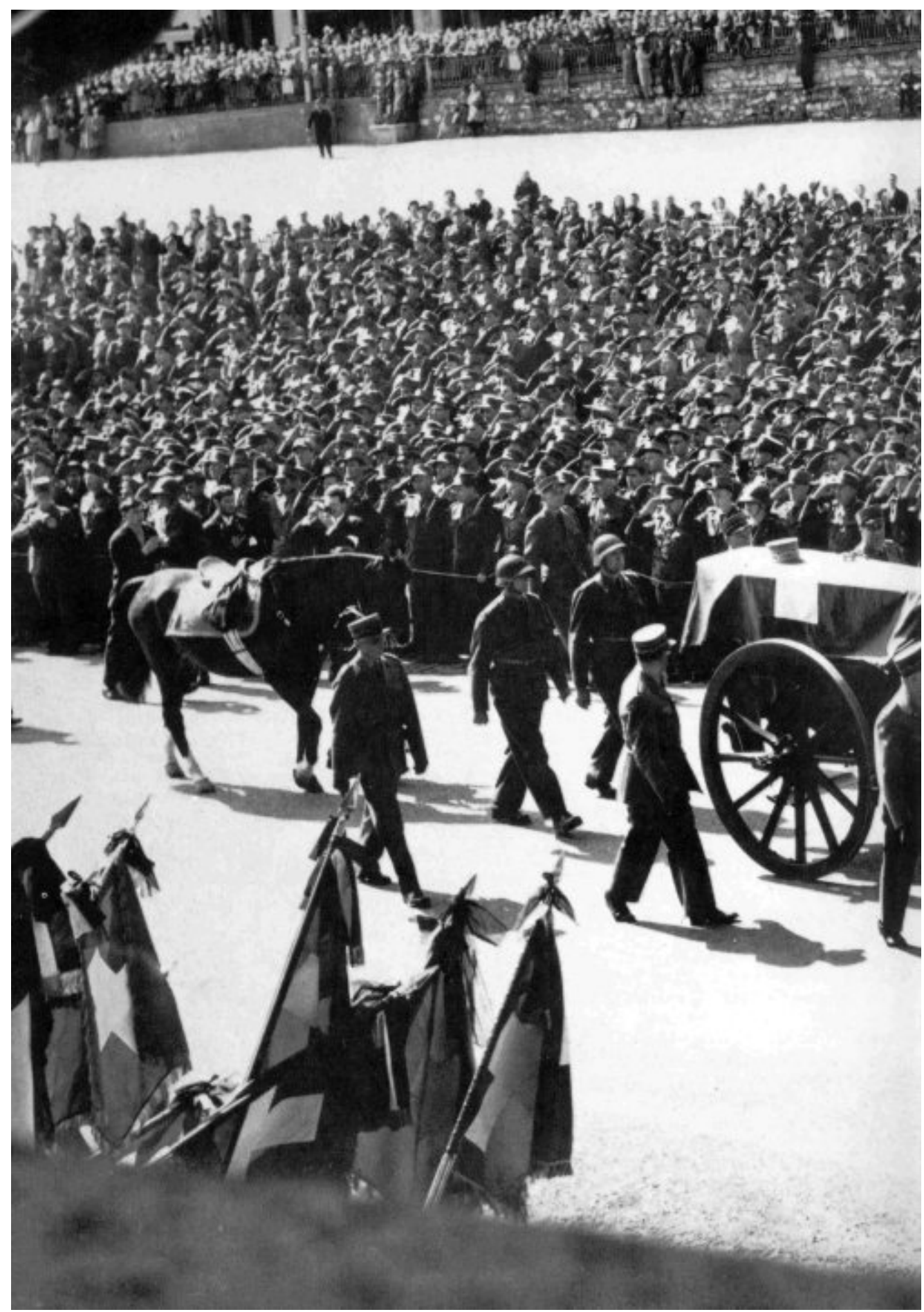

o Pully, Centre général Guisan

Bien plus que le monument équestre coulé dans le bronze, l'ampleur des funérailles du général (fig. 13) contribuera à alimenter la légende de Guisan. De par leur faste et leur couverture médiatique, ces obsèques dépassent de très loin les hommages traditionnellement rendus à un chef militaire ou un homme politique en Suisse. Une image restera d'ailleurs présente dans la 
mémoire collective : celle du cheval noir du général suivant, lors du cortège funèbre dans les rues de la capitale vaudoise, le cercueil de son maître posé sur un affût d'artillerie.

\section{Notes}

1 Un dossier sur cette polémique se trouve au Centre d'histoire et de prospective militaires à Verte Rive (Pully/Lausanne) ; les différentes informations concernant le monument en sont issues. Nous remercions M. Antoine Schulé pour nous l'avoir aimablement mis à disposition. 2 [Villard], « Pauvre général, pauvre cheval ! », Le Courrier, 28 juillet 1966.

3 Neue Zürcher Zeitung, 3 juin 1967 [traduction par François Vallotton].

4 On signalera toutefois l'exception constituée par le portrait équestre de François-Pierre König von Mohr, réalisé par Samuel Hofmann en 1631. Considéré comme le premier portrait équestre dans l'histoire de la peinture suisse, celui-ci se distingue tant par ses dimensions que par la posture princière du personnage, un officier fribourgeois qui vient d'être anobli par l'Empereur Ferdinand. Nous remercions Jean Steinauer, auteur d'un livre sur König, pour ces informations et renvoyons au récent ouvrage collectif, Les chevauchées du colonel Koenig : un aventurier dans l'Europe en guerre, 1594-1647, Fribourg : Faim de siècle, 2006.

5 Hervé de Weck, La cavalerie à travers les âges, Lausanne : Edita, 1980, pp. 220-224.

6 Walter Lietke, The Royal Horse and Rider. Painting, Sculpture and Horsemanship 1500-1800, New York, Metropolitan Museum of Art, Abaris Books, 1989 ; Janson, Horst W., "The Equestrian Monument from Cangrande della Scala to Peter the Great", Aspects of the Renaissance, Austin, London, 1967, p. 73-85; Le cheval et la guerre du XV au XX siècle, sous la direction de Daniel Roche, Paris : Association pour l'académie d'art équestre de Versailles, 2002.

7 Voir les biographies et l'iconographie dans Charles Gos, Généraux suisse commandants en chef de l'armée suisse de Marignan à 1914, préface du colonel commandant de corps Guisan, Neuchâtel : Attinger, 1932 (réimprimé par Cabédita en 1990).

8 Sur Dufour et son image, voir : G. H. Dufour. L'homme, l'œuvre, la légende. Le portrait topographique de la Suisse, Genève : Musée d'art et d'histoire, Musée de Carouge, 1987 ; Philippe Kaenel (éd.), 1848 ; le carrefour suisse. Le pouvoir des images, Lausanne : Ed. Payot, 1998 (sur Dufour voir Philippe Kaenel, « Le général, le conseiller fédéral et les réfugiés : types politiques et sociaux », p. 133-150). Voir également, dans un contexte différent, les problèmes posés par le portrait équestre monumental, héroïque et «sublime » du duc de Wellington, conçu par Matthew Cotes Wyatt pour Hyde Park Corner en 1846, et dont les dimensions et l'iconographie ne correspondaient pas à l'image de « britishness » (honnêteté, patience, fidélité...) du personnage : P. W. Sinnema, «Wyatt's Wellington » and the Hyde Park Corner Controversy », The Oxford Art Journal, 27 février 2004, p. 173-192.

9 Cité dans Olivier Reverdin, La guerre du Sonderbund vue par le général Dufour juin 1847 - avril 1848, d'après des lettres et des documents inédits, Genève : Éditions du Journal de Genève, 1948, p. 103.

10 Lettre du 28 décembre 1847, citée dans Reverdin 1948, p. 103-104.

11 Lettre du 28 décembre 1847, citée dans Reverdin 1948, p. 84.

12 Lettre de décembre 1847, citée dans Reverdin 1948, p. 103.

13 Sur la cavalerie suisse, voir notamment l'apologie de Robert Staub, Cavalerie immortelle, Lausanne : Sillon romand, 1948, ainsi que Max E. Ammann, Le Fédéral. Trois siècles de cavalerie suisse, Lausanne : Bibliothèque des arts, 1975. Les écrits de Wille sur l'armée et la cavalerie sont réunis dans Edgar Schumacher (éd.,), General Ulrich Wille, Gesammelte Schriften, Zurich : Freze Verlag, 1941.

14 Voir Rudolf Jaun, Preussen vor Augen. Das schweizerische Offizierskorps im militärischen und gesellschaftlichen Wandel des Fin de siècle, Zurich : Chronos, 1999.

15 Ibid., p. 342-344.

$16 \mathrm{La}$ littérature secondaire s'étend des biographies partisanes (notamment celles d'Edgar Schumacher en 1940, de Carl Helbing en 1956, de Heinz Christian Röthlisberger en 1975) aux approches violemment critiques, principalement le best-seller de Niklaus Meienberg, Die 
Welt als Wille und Wahn. Elemente zur Naturgeschichte eines Clans, Zurich : Limmat Verlag, 1987 (trad. : Le délire général. L’armée suisse sous influence, Genève, Zoé, 1988). En 2003, un colloque intitulé General Ulrich Wille. Vorbild den einen - Feindbild den Anderen (éd. par Hans Rudoph Fuhrer et Paul Maeinrad, Zurich, Verlag Neue Zürcher Zeitung) s'est donné pour tâche de confronter divers points de vue.

17 Sur les relations entre Hodler et Wille, voir Jura Brüschweiler, Ferdinand Hodler porträtiert General Wille, Zurich : Kunsthaus, 1975 et du même : Hodler, Martigny : Fondation Giannada, 1991.

18 La carte postale est l'imagerie mass médiatique par excellence autour de 1900. En effet, vers 1902, la Suisse détient le record mondial d'envoi de cartes postales par tête d'habitant, soit 22 millions de cartes dont 8 millions de fabrication suisse (voir Otto Wicki, Geschichte der Post-und Ansichtskarten, Berne : Bibliothèque nationale, 1996, p. 18).

19 Voir, sous la plume d'un certain «Firmin », l'article « Têtes de chefs » sur le « général Vuille » dans L'Arbalète $\mathrm{n}^{\circ} 18,1917$ : «L'Helvète rabougri. - Gros et court, en effet, et gros de partout, court de partout. On pense à des boudins de différents calibres, amoncelés. // Courte et grosse, la lèvre inférieure, et de même galbe que la ligne inférieure des yeux. Lèvres et yeux débordent à l'angle extérieur ; la ligne ne monte qu'à regret pour clore l'organe. Un psychologue y verrait un signe d'épaississement moral. // Comment est-il devenu général ? Il est si peu militaire. Je le vois en civil, des petits enfants autour de lui, qui lui grimpent aux genoux (...). Mais qui ou quoi l'a fait général ? - Est-il un chef ? Avec cet air paternel... Est-ce une intelligence ? Pas d'éclair napoléonien dans ces gros yeux. Une volonté ? je ne vois de viril que son nom déformé, prononcé à la germanique. (...) Suisse, oui, il l'est ; plus que Suisse même : on dirait le Parti Radical en uniforme !».

20 Artiste lausannois, Gea Augsbourg, de son vrai nom Georges-Charles Augsburger (1902-1974), a collaboré comme illustrateur à de nombreux ouvrages et s'est fait connaître comme dessinateur et caricaturiste dans des périodiques tant français (Vendredi, Paris-Soir, Les Nouvelles littéraires, La Tribune des Nations) que suisses (Le Radio, Formes et couleurs, Servir, Contacts).

21 Baudin, Antoine, Géa Augsbourg 1902-1974, Lausanne : En bas, 2002, p. 76.

22 Le Ciné-journal suisse n²47 (« Une journée au PC du Général »), 6 juillet 1945.

23 Cette composante se retrouve notamment chez Mussolini dont les postures équestres ont été analysées par Sergio Luzzatto, «L'immagine del Duce. Mussolini nelle fotografie dell'Istituto Luce », in Les portraits du pouvoir, Actes du colloque organisé par Olivier Bonfait et Brigitte Marin, Paris ; Rome, Académie de France ; Somogy, 2003, p. 159-175.

24 Willi Gautschi, Le Général Guisan. Le commandement de l'armée suisse pendant la Seconde Guerre mondiale, Lausanne : Payot, 1991 (1989 pour l'édition allemande), p. 160. 25 Ibid., p. 556.

26 Quelques pièces de correspondance sur ce dossier se trouvent au Cabinet des estampes de la Bibliothèque Nationale, sous la cote 1941. G 784. Nous remercions Madame Susanne Bieri pour la mise à disposition de ces documents.

27 Sur la révision de l'image de Guisan dans l'historiographie suisse, voir, outre la biographie de Gautschi, Erwin Bucher, Zwischen Bundesrat und General : Schweizer Politik und Armee im Zweiten Weltkrieg, Sankt Gallen : Verlagsgemeinschaft St. Gallen, 1991 ; Hans Ulrich Jost, Le salaire des neutres, Paris : Denoël, 1999.

28 Voir plus haut note 11.

29 Lettre de André Weissbrodt, appointé chauffeur-militaire, Lausanne, 30 mars 1966 ; elle figure dans le dossier concernant le Comité monument Général Guisan mentionné dans la note 1 .

\section{Pour citer cet article}

Référence électronique 
Philippe Kaenel et François Vallotton, «Le général et son cheval : figures du pouvoir militaire en démocratie, à l'exemple de la Suisse », Bulletin du Centre de recherche du château de Versailles [En ligne], Objets et insignes du Pouvoir, mis en ligne le 20 février 2008. URL : http://crcv.revues.org/114

\section{À propos des auteurs}

\section{Philippe Kaenel}

Philippe Kaenel, professeur suppléant d'histoire de l'art, maître d'enseignement et de recherche à l'université de Lausanne, est spécialisé dans le domaine des arts graphiques et photographiques (actuellement, il rédige une histoire de la caricature en Suisse de la Renaissance à nos jours). Sa thèse, soutenue à l'université de Lausanne, porte sur Le métier d'illustrateur 1800-1830. Rodolphe Töpffer, J.-J. Grandville, Gustave Doré (Paris, 1995, réédition en poche chez Droz en 2005).

\section{François Vallotton}

François Vallotton est professeur assistant d'histoire contemporaine à l'université de Lausanne où il enseigne plus spécialement l'histoire des médias. Auteur de nombreuses contributions sur l'histoire culturelle et intellectuelle de la Suisse, il a notamment consacré sa thèse à l'histoire de l'édition suisse francophone (L'édition romande et ses acteurs 1850-1920, Genève : Slatkine, 2001) et travaille parallèlement au développement de la Fondation Mémoire Éditoriale qui a pour vocation la préservation et la mise en valeur des archives éditoriales en Suisse.

\section{Droits d'auteur}

(c) Tous droits réservés

\section{Résumé}

Dans le cadre d'un séminaire interdisciplinaire en histoire et histoire de l'art à l'université de Lausanne, nous avons travaillé sur les «cultures de guerre » en Suisse et en Europe durant la première moitié $\mathrm{du} \mathrm{xx}^{\mathrm{e}}$ siècle. En nous interrogeant notamment sur le caractère singulier ou non de celles-ci dans un pays dont le territoire n'a pas connu d'opérations militaires d'envergure. Les généraux Ulrich Wille et Henri Guisan sont deux figures incontournables du champ médiatique et du champ militaire helvétique. Outre leur accession à la tête de l'armée suisse durant les deux conflits mondiaux, Wille et Guisan sont au cœur des représentations d'une institution amenée à jouer un rôle idéologique inversement proportionnel à sa fonction stratégique et militaire. La représentation du général renvoie à une série de schèmes iconographiques, de l'Antiquité à la Galerie des batailles du château de Versailles. Comment le cas suisse s'articule -t -il sur cette histoire et à quelles fonctions obéissent les représentations de Wille et Guisan dans leur contexte d'émergence ? Comment les généraux d'un pays neutre, c'est-à-dire non belligérant, ont-ils trouvé une posture qui corresponde à leur statut militaire très particulier et comment ont-ils imaginé leurs relations avec l'autorité politique ? Le rôle emblématique du cheval dans les deux guerres mondiales et les fonctions symboliques du portrait équestre comme figure du pouvoir militaire se placent au cœur de cette réflexion entre histoire et histoire de l'art.

Mots clés : Général, portrait équestre, armée, censure, Suisse, Première Guerre mondiale, GuillaumeHenri Dufour, Ulrich Wille, Henri Guisan, caricature 